\title{
Conocimientos, percepciones y prácticas relacionados a equinococosis quística en familias con antecedentes de la enfermedad
}

\author{
KNOWLEDGE, PERCEPTIONS AND PRACTICES RELATED TO CYSTIC ECHINOCOCCOSIS IN \\ FAMILIES WITH HISTORY OF THIS ILLNESS \\ Yelina Ramírez D. ${ }^{1}$, Elisa Jefferson M.², Henry Hernández I. ${ }^{3}$, Daphne León C. ${ }^{1}$, \\ Néstor Falcón P. ${ }^{1,4}$
}

\section{RESUMEN}

El objetivo del estudio fue sistematizar la información acerca del conocimiento, percepciones y prácticas asociadas a la exposición a Echinococcus granulosus en familias con antecedentes de equinococosis quística (EQ) de la provincia de Huancayo, región Junín (Perú), que fueron atendidos en el Instituto Nacional de Salud del Niño durante el periodo 2004-2012. Se elaboró una guía de entrevista que se aplicó al miembro de la familia más cercana al caso del paciente con EQ, pero permitiéndose la intervención de los demás miembros. Extractos de la transcripción de los audios fueron categorizadas como factores de riesgo para contraer la enfermedad, impactos sobre el paciente y la familia, y cambios producidos en el entorno pos-enfermedad. La sección cuantitativa resumió información en base a estadística descriptiva. Se demostró la persistencia de deficiencias en cuanto a conocimientos, percepciones y prácticas de los entrevistados y sus familias respecto a la EQ. Las familias mantienen hábitos y prácticas que favorecen la transmisión y diseminación de la enfermedad y la experiencia de sufrir la enfermedad años atrás no produjo cambios positivos en sus estilos de vida. Se espera que los resultados sirvan como antecedentes para actuar frente a los aspectos sociales de esta enfermedad.

Palabras clave: equinococosis; quiste hidático; hidatidosis; zoonosis

\section{AbSTRaCT}

The aim of this study was to systematize information about the knowledge, perceptions and practices associated with exposure to Echinococcus granulosus in

1. Grupo de Salud Pública Veterinaria (SAPUVET-Perú), Facultad de Medicina Veterinaria y Zootecnia, ${ }^{2}$ Facultad de Psicología, Universidad Peruana Cayetano Heredia, Lima, Perú

${ }^{3}$ Instituto Nacional de Salud del Niño, Ministerio de Salud, Lima, Perú

${ }^{4}$ E-mail: nestorfalcon@upch.pe

Recibido: 13 de junio de 2017

Aceptado para publicación: 30 de octubre de 2017 
families with a history of cystic echinococcosis (EQ) from the province of Huancayo, Junín region (Peru), attended at the National Institute of Child Health during the period 20042012. An interview guide was developed and applied to the family member closest to the case of the patient with EQ, but allowing the intervention of other members. Extracts from the transcription of the audios were categorized as risk factors for contracting the disease, impacts on the patient and the family, and change produced in the post disease environment. The quantitative section was summarized on descriptive statistics. It was demonstrated the persistence of deficiencies in the knowledge, perceptions and practices of the interviewees and their families regarding the EQ. Families maintain habits and practices that favour the transmission and dissemination of the disease. The experience of suffering the disease years ago has not produced positive changes in their lifestyles. It is hoped that the results serve as antecedents to act against the social aspects of this disease.

Key words: echinococcosis; hydatic cystic; hydatidosis; zoonoses

\section{INTRODUCCIÓN}

La equinococosis quística (EQ) o hidatidosis es una zoonosis globalmente distribuida. En Sudamérica, Argentina, Brasil, Chile, Perú y Uruguay son considerados altamente endémicos (Mandal S y Mandal M, 2012). En el Perú, las tasas de incidencia acumulada fluctúan entre 39 y 79 casos por cada 100000 habitantes. Sin embargo, desde el abandono en 1980 del Proyecto de Control Piloto de Hidatidosis en la Sierra Central del Perú (Pérez, 2007), la hidatidosis no es atendida por el estado y es una de las enfermedades postergadas (Moro et al., 1997; Rafael et al., 2008; Irabedra y Salvatella, 2010).

En estudios realizados en animales en el departamento de Junín se reporta $87 \%$ de prevalencia en ovinos y entre 32 y $79 \%$ en perros, siendo estas las prevalencias reportadas más altas de América del Sur (Cardona y Carmena, 2013; Carmena y Cardona, 2013).

La EQ ocasiona grandes pérdidas económicas debido a los altos costos de internamiento y tratamiento de las personas, que en algunos casos requieren de interven- ciones quirúrgicas. En un hospital de niños en Lima, Perú, se estimó entre 2006 y 2010 un costo de US\$ 914 y 298 para la atención de pacientes hospitalizados con y sin cirugía, respectivamente (Fano et al., 2014). En la parte veterinaria de animales de producción, la enfermedad también produce elevadas pérdidas en función del valor de las vísceras decomisadas y por mermas en la producción de lana, leche y carne (Venegas et al., 2014).

La EQ es producida por el Echinococcus granulosus, que tiene como principal hospedero intermediario a las ovejas y como hospedero definitivo a los perros (Mandal S y Mandal M, 2012). La perpetuación del ciclo de vida del E. granulosus depende de la convivencia de hospederos definitivos e intermediarios y ciertos hábitos de las personas, como el suministro de vísceras a los perros como alimento, el contacto cercano con perros, consumo de agua contaminada o productos frescos no lavados (frutas y hortalizas), así como la ausencia de un control veterinario para la desparasitación de canes y control del beneficio de los hospederos intermediarios (Larrieu et al., 2004; Moro et al., 2008; Santivañez et al., 2010) e ignorancia de las normas de higiene (Larrieu et al., 2004). 
La estrategia base para el control de la hidatidosis apuntaría a la interrupción del ciclo biológico del E. granulosus en la cadena epidemiológica de áreas endémicas. La ausencia de un programa integral de control de esta enfermedad se considera como la principal causa del incremento de la prevalencia de equinococosis humana en el Perú (Moro et al., 1997; Irabedra y Salvatella, 2010). En países como Argentina, Chile y Uruguay, que mantienen medidas de control basadas en la desparasitación de perros con praziquantel cada 45 días, legislación para regular la población canina, control de la faena en mataderos oficiales y educación sanitaria, se ha observado la disminución de la prevalencia e incidencia de hidatidosis humana y animal (Jiménez et al., 2004; Irabedra y Salvatella, 2010; Larrieu y Zanini, 2012; Mandal S y Mandal M, 2012). Sin embargo, muchos de estos programas no han sido mantenidos en el tiempo y la tasa de reinfección es alta (Larrieu y Zanini, 2012).

Debido a la importancia de esta enfermedad en la salud pública y al rol protagonista que tiene el ser humano para el cumplimiento del ciclo de transmisión, se requiere comprender el entorno cultural de las personas afectadas por EQ y determinar el nivel de conocimientos que tienen al respecto. Por ello, el objetivo de este estudio fue determinar conocimiento, percepciones y prácticas asociadas a la exposición a E. granulosus en familias con antecedentes de EQ en la provincia de Huancayo, región Junín, Perú.

\section{Materiales y Métodos}

El estudio cuali-cuantitativo se desarrolló en la provincia de Huancayo, localizada en la Sierra Central del Perú. La información de pacientes con antecedentes de EQ se obtuvo en la Oficina de Epidemiología de la Estrategia Sanitaria de Zoonosis del Instituto Nacional de Salud del Niño (INSN), Lima. La sistematización de la información se realizó en la Facultad de Medicina Veterinaria y Zootecnia de la Universidad Peruana Cayetano Heredia (FAVEZ-UPCH).

La población objetivo estuvo constituida por las familias de pacientes diagnosticados con EQ humana de la provincia de Huancayo, atendidos en el INSN durante el periodo 2004-2012. Se obtuvieron 27 historias clínicas con dichas características. Se hizo seguimiento de los pacientes y tras haber solicitado la aceptación para participar en el estudio mediante la firma de un consentimiento informado se logró reclutar a 15 familias.

Se elaboró una guía de entrevista donde se incluyeron las preguntas base que fueron formuladas a las familias. Se consideró información demográfica, conocimientos acerca de EQ, modo de transmisión, medidas de prevención e impacto de la enfermedad.

Las entrevistas se realizaron en fecha convenida y en las viviendas de los participantes. Se consideró como el principal entrevistado al miembro de la familia que acompañó al paciente con EQ durante todo el proceso de la enfermedad; asimismo, se permitió la intervención de los demás miembros de la familia sin ninguna restricción. Las entrevistas fueron grabadas.

Para el análisis de la información se transcribieron todos los audios de voz a un documento en formato Word. Se sistematizó la información empezando con la lectura crítica de las transcripciones, extrayendo las expresiones de los entrevistados respecto a sus vivencias en el entorno de la EQ, las que fueron categorizadas como factores de riesgo para contraer la enfermedad, impactos sobre el paciente y la familia, y cambios producidos en el entorno pos-enfermedad. Estas expresiones buscaron reflejar los sentimientos y vivencias en el entorno de la familia debido a la enfermedad sufrida por el paciente. 
Todos los procedimientos realizados para la obtención de información estuvieron centrados en preservar la integridad y los derechos fundamentales de los participantes en la investigación. El estudio fue aprobado por el Comité Institucional de Ética de la $\mathrm{UPCH}$

\section{Resultados}

El estudio recuperó información de 15 familias con antecedentes de pacientes con EQ. En el Cuadro 1 se presenta la información general de los entrevistados. La mayor frecuencia de casos se encontró en el estrato etario de 5 a 10 años, el primer signo clínico reportado como alarma de enfermedad fue la tos, y el tiempo que transcurrió entre la presentación del signo y la atención médica fue desde menos de un mes hasta seis meses en la mayoría de los casos.

El número de quistes encontrados con más frecuencia fue de $1 \circ 2$, siendo el pulmón la ubicación anatómica más frecuente (Cuadro 2).

El 80\% (12/15) de los entrevistados mencionó que los niños no tuvieron secuelas tras la intervención contra la EQ. En los demás, la secuela fue la muerte, escoliosis o intolerancia al ejercicio físico. En cuanto al control anual que fuera recomendado tras la intervención, el 66.7\% (10/15) no lo realizó, el 20\% (3/15) lo realizó de forma incompleta (dos pacientes realizaron solo un control, otro realizó dos controles y después lo abandonó). Solo el 13.3\% (2/15) venía cumpliendo con el control periódico anual hasta el momento de la entrevista.

Antes de vivir la experiencia de un paciente con EQ en el entorno familiar, todos los entrevistados desconocían la enfermedad y su forma de transmisión. Después de haber sufrido la experiencia, solo el $13.3 \%(2 / 15)$ tenía claro el ciclo de transmisión de la enfermedad.
En los cuadros 3 al 6 se resumen las expresiones de los entrevistados respecto a sus vivencias en el entorno de la EQH humana.

\section{Discusión}

El estudio muestra el impacto de la EQ sobre el paciente y entorno familiar de los niños que han sufrido esta enfermedad. La mayoría de entrevistados fueron amas de casa debido a que ellas son las personas que suelen encontrarse en el hogar. Además, ellas fueron quienes estuvieron al lado de los niños durante el periodo de enfermedad y recuperación. Todos provenían de zonas endémicas a EQ, documentadas en investigaciones previas (Pérez, 2007; Rojas; 2012; Zúñiga, 2014). La mayoría de las familias tenía más de un hijo por lo que la enfermedad de uno de ellos representó el descuido en la atención de los otros.

La mayoría de los pacientes tenían menos de 10 años cuando presentaron los primeros signos clínicos. Esto indica que la infección se produjo a temprana edad, donde la exposición con huevos de E. granulosus es más fácil debido, entre otras razones, a un mayor contacto del niño con los animales y con ambientes contaminados. Aybar et al. (2012) mencionan que la mayor frecuencia de casos autóctonos de EQ en Lima se encontró en niños de 5 a 9 años.

El mayor número de quistes hidatídicos diagnosticados fue entre 1 y 2 . En contraste, Velarde (2002) encontró un solo quiste en el $85.7 \%$ de pacientes con EQ, $13.7 \%$ con dos quistes y solo el $0.6 \%$ de pacientes con tres quistes hidatídicos en el Hospital Arzobispo Loayza de Lima. La principal ubicación anatómica del quiste fue el pulmón; probablemente debido a que la mayoría de pacientes llegó al hospital con signos clínicos compatibles con enfermedades pulmonares. Esto difiere a lo mencionado por Acha y Szyfres (2003), quienes refieren que la localización más frecuente es el hígado con $65-70 \%$ de los casos, segui- 
Cuadro 1. Información demográfica de personas entrevistadas con antecedentes de hijos con equinococosis quística en la región Junín, Perú

\begin{tabular}{llcc}
\hline Variable & Estrato de la variable & Número & Porcentaje \\
\hline Ocupación del entrevistado & Ama de casa & 10 & 66.7 \\
& Comerciante & 4 & 26.7 \\
& Agricultor & 1 & 6.6 \\
Procedencia del entrevistado & Junín & 9 & 60.0 \\
& Huancavelica & 4 & 26.6 \\
& Lima & 1 & 6.7 \\
& Puno & 1 & 6.7 \\
Número de hijos & 1 & 3 & 20.0 \\
& 2 & 4 & 26.7 \\
& 3 & 2 & 13.3 \\
& 4 & 4 & 26.7 \\
& $>4$ & 2 & 13.3 \\
\hline
\end{tabular}

Cuadro 2. Información referida por el entrevistado acerca del niño afectado con equinococosis quística humana. Región Junín - Perú

\begin{tabular}{llcc}
\hline Variable & Estrato de la variable & Número & Porcentaje \\
\hline Edad del paciente a la & Menor a 5 años & 3 & 20.0 \\
presentación del primer signo & De 5 a 10 años & 8 & 53.3 \\
clínico & Más de 10 años & 4 & 26.7 \\
Número de quistes & 1 & 6 & 40.0 \\
diagnosticados & 2 & 6 & 40.0 \\
& 3 & 2 & 13.3 \\
\multirow{2}{*}{ Ubicación anatómica de los } & 6 & 1 & 6.7 \\
quistes & Pulmón & 8 & 53.2 \\
& Pulmón / hígado & 3 & 20.0 \\
& Pulmón / peritoneal & 1 & 6.7 \\
& Hígado / cerebro & 1 & 6.7 \\
& Hígado & 1 & 6.7 \\
& Cerebro & 1 & 6.7 \\
\hline
\end{tabular}

do de los pulmones con $25 \%$, y que en raras ocasiones pueden ubicarse en órganos como riñón, bazo, huesos y cerebro. Esto es debido a la ruta sanguínea que toma el embrión hexacanto, que después de atravesar el intestino delgado, llega al hígado y, posteriormente, a los pulmones a través de la arteria pulmonar (Rubio et al., 2012).
La persistencia del E. granulosus se ve favorecida por una serie de factores, en los que el humano juega un rol importante. Muchos de estos persisten debido al desconocimiento del ciclo biológico, las creencias y prácticas equívocas, que da como resultado afecciones, muchas veces irreversibles, 
Cuadro 3. Expresiones de los entrevistados respecto a vivencias relacionadas a factores de riesgos para la presentación de equinococosis quística humana en la región Junín (Perú)

Alimentación de los perros

- "Esas vísceras cuando están contaminadas como te digo esa parte nomás lo sacamos y le damos al perro. Así hacíamos, pero dice debemos enterrarlo. Pero acá no hacen eso. No es costumbre" (E001).

- "Mi abuelita decía que era bazo (por el quiste hidatídico)...mi abuelita le daba a los perros" (E008).

Desparasitación de canes

- "En el campo si habia perros. Esos seis meses no he visto que desparasiten sus perros" (E006).

Exposición a huevos de E. granulosus

- "Mi hijita jugaba con los perros, aunque se les dice se laven (las manos) y ahi están jugando y después ya comen y no se lavan” (E014).

- "Tenía (el niño) la costumbre de besar en la boca a los perros" (E015).

Hábitos de higiene

- "Se va a jugar, con su mano sucia llega, no se lava y quiere comer. Como niña se le tiene que decir que se lave. Yo le digo, pero él me dice eso no hace nada" (E007).

- "Yo me acercaba al perro, me gusta mucho a mí...no me lavaba las manos después de jugar" (E010 - el niño).

Conocimiento de la forma de transmisión de la enfermedad

- "Has criado tus gallinas junto con tus perros y eso te lo has hecho comer a tu hijo. Eso me dijeron los doctores...no se la verdad cual es la causa original" (E002).

- "Es mediante las heces del chancho, carnero. Eso dice cuando lo agarra, no lo presenta al instante sino de acá a un largo tiempo en años...o sino comer infectado el hígado del carnero o de cualquier animal" (E006).

Conocimiento de la forma de prevenir la enfermedad

- "Lo único que yo sé, es no tener nada de animales, ni gato, ni perro, ni nada. Y cocinar bien" (E015).

- “No se señorita, no sé cómo se evitaría, ¿ser limpio?” (E010).

La enfermedad en el entorno de la familia

- "Acá tengo una vecina que tiene bastantes perros, y una vez a su hija le operaron de quistes en los pulmones" (E012).

- "Había un joven que tenía quiste, y hubo campeonato de interbarrios, y el balón le cayó en la espalda, como estaría apenas de reventar, se reventó y murió" (E005).

sobre la salud de las personas. Algunos estudios indican que incluso personas que se encuentran más expuestas laboralmente realizan prácticas erróneas, como la alimentación de perros con vísceras (Reyes et al., 2012; Merino et al., 2017), por lo que se puede deducir que esta problemática se acentúa en la población general.
En esta investigación, los entrevistados mencionaron que en su entorno familiar y local persiste el hábito de alimentar a los perros con vísceras, especialmente las infectadas, debido a que estas no son consumidas por ellos. Además, se puede decir que la desparasitación periódica de sus mascotas no 
Cuadro 4. Expresiones de los entrevistados respecto a vivencias relacionadas a la salud del paciente afectado por equinococosis quística humana y sus familiares en la Región Junín (Perú)

La salud del paciente

- "Ella estaba yendo bien a sus clases, pero me dijo mamá que me estará pasando. Estoy viendo a mi profesora el doble, mi letra también estoy viendo al doble, no sé qué me está pasando mami” (E008).

- "...hasta que ella ya sentía más peor, ya su bracito se adormeció...mami mi brazo ya no quiere alzarse, aqui me molesta” (E014).

- "Ya estaba más grave porque cuando toseaba (tosía) ya empezó a botar sangre; así que le dijimos ya por emergencia hay que llevarle, y la fiebre ya estaba en 40...desde ahi se quedó internado" (E015).

- “...él seguía empeorando porque el dolor de cabeza era más, y se estreñía más. Mi hijito lloraba, y cuando se enfermaba de la tos no quería ni que le toquemos, le dolería supongo" (E012).

- “...lloraba de dolor, no le podían dejar. Le compraban otros medicamentos para que calme el dolor...después de la operación permaneció dos meses en el hospital" (E001).

- "No tenía buen físico, no podía correr tanto y me agitaba muy rápido...vomitaba y decía ayúdame yo quiero vivir, ya no podía caminar bien, le llevaba así como un trapito... al momento de despedirse para la operación mi hijo se puso a llorar..." (E006).

La salud del familiar

- "Aquí pues señorita recordándome de mi chiquita, no puedo olvidar...no hablaba ni se movía, igualito estaba...estaba despierta me dice el doctor, háblale a ver, yo le hablo le digo hijita como estás y ella empezó a llorar, escuchaba, empezó a llorar yo también me he llorado señorita, papá donde te has ido, donde me has dejado dirá" (E008).

- "Era bien triste todo lo que hemos pasado..., todos tenían pena. Bien triste es estar todos los días en el hospital..., como mi casa era el hospital, me deprimía” (E001).

- "Señorita es terrible pasar eso...yo soy bien fuerte, fuerte, nunca he llorado con mis hijos, pero como me dicen que puede llegar hasta el cerebro (el quiste pulmonar habia reventado)...yo quisiera que mis hijos estén sanos, sean profesionales" (E014).

- "No entendia bien por todo lo que estaba pasando, tenía pena de mi hijo, de mi hijita también tenía pena. Mi vida era total pena...llorar era mi vida, porque solita estaba, nadie estaba" (E010).

- “...una cosa que me chocó bastante fue el ver a mi hijo después de la operación, me afectó bastante a mi más que nada, estuve ahi día y noche con él" (E009).

- "Emocionalmente me afectó, yo comencé a adelgazar como le veía mal, botaba sangre, me desesperaba porque los doctores me decían que no va a poder ser internado" (E015). 
Cuadro 5. Expresiones de los entrevistados respecto a vivencias producidas durante la búsqueda de la mejora de salud del paciente afectado por equinococosis quística humana en la Región Junín (Perú)

Búsqueda de soluciones no convencionales

- "Unas personas me dijeron que hay un naturista que ha estudiado en Cuba, él le va a curar, me dijeron personas que él les había sanado pero de otras enfermedades. Entonces le busqué, decía aunque sea de poco en poco le voy a pagar, porque cuanto me va a costar la operación" (E001).

- "Yo he ido donde el curandero, adivinadores, me han dicho será daño y le he llevado. Después nada ha hecho, no ha sanado" (E008).

Alejamiento del hogar

- "...le hice operar solita a mi hijo y he estado sola con él, a mi hijita le dejé a mi suegra y con él nomás me fui para Lima, y en Lima ha estado casi un mes internado" (E010).

- "Yo pagué mis pasajes, y como mi sobrina vive en Lima me dieron alojamiento, me apoyaron. Estuve casi medio año" (E003).

Impacto sobre la economía familiar

- "Mi esposo trabajaba y toda la platita me enviaba" (E001).

- "Lo económico también bastante ha incumbido, fue dificil de salir de esto. Estaba sola todo el tiempo, tuve que enfrentar para pagar el préstamo" (E002).

Temores respecto a la salud del niño post curación

- "Yo estaba preocupada, si va a estar bien, si habrá aumentado, bajado (tamaño del quiste)" (E004).

- "Me da un poco de temor cuando voy al hospital y sacamos la resonancia, para ver si tiene o no tiene" (E012).

La relación con los profesionales de la salud

- “...pero tenía que rogarles a la señorita (por la transferencia a Lima), porque dice que las personas que van dice a Lima no tienen donde quedarse, y por eso decían que tenías que tener un familiar allá. Yo le rogué le dije aunque sea voy a quedarme en la calle, yo quiero que mi hijo se sane" (E015).

- "La doctora me dijo tu hijo se va a morir. Por eso digo la misma ética, debió tratar de explicarme, decirme las cosas con calma, pero no ella me lo soltó así, tu hijo se va a morir. Aquí lo único que tienen que hacerle es operarle, pero no creo que se salve, así me dijo, te juro que me he enfermado me he ido al estrés, la depresión, he estado mal por 4 años" (E013).

es usual, exponiéndolos a la equinococosis. Merino et al. (2017) mencionan que la exposición a E. granulosus para las personas aumenta al permitir que sus mascotas defequen en sus casas o alrededores, lugares en los que permanecen o juegan los niños. Esta exposición incrementa cuando los entrevistados reconocen que los niños «besaban» a los perros en el hocico y que sus hábitos de higiene eran deficientes.

El estudio muestra que la experiencia de haber tenido un miembro de la familia con EQ no ha mejorado sus conocimientos sobre 
Cuadro 6. Expresiones de los entrevistados respecto a vivencias producidas posteriores a la enfermedad de los pacientes afectados por equinococosis quística humana en la Región Junín (Perú)

Control del paciente

- "El doctor me dijo que le haga un chequeo, pero no le hemos hecho, ya han pasado 3 años ... aqui fui al hospital y me dijeron que le saque radiografia, pero como estaba estudiando no le saque y lo deje" (E001).

- "Ahora tiene 15, han pasado 5 años aproximadamente y ningún control porque le veo sana, porque hay veces también es plata pues señorita" (E011).

Comportamiento del paciente

- "Le gusta jugar básquet, le gusta hacer barra, le gusta hacer plancha, porque él es libre dice ahora. Esas cosas no hacía antes" (E006).

- “...pero también le generé una malcriadez ya porque le permitía hacer demasiadas cosas, ya no le ponía límites, me daba pena cualquier cosa que le podía pasar" (E009).

En los hábitos de consumo de alimento

- "El hígado ya no comemos. No come mucha carne. El doctor nos ha dicho que la carne está contaminada que no deben comer... a mi me gusta el higado, pero ellos no comen por temor" (E004).

- “... a mi hijito le han prohibido que coma carne y mucho menos las vísceras. Solamente le doy pollo, pavita y pescado. Carne roja no mucho. Nosotros tampoco comemos ya nos da miedo" (E012).

Temor a la crianza de los animales

- "Yo te juro que desde que se ha enfermado a todo perro le tenía cólera, no podía ni verlo. ¡Desde lejos nomas! De verdad” (E013).

- "Por eso ya no quisiera que ni a mi hija, que nadie le pase eso. Tengo temor, yo ya no crio animales" (E015).

Cambio en los hábitos de higiene

- “...ahora ya le estoy lavando las manos, salimos de cualquier parte, o si no me llevo mi botellita de agua porque siempre ellos están corriendo por el pasto y siempre hay heces de perro, asi que estamos tomando esa precaución. Cada que come o juega le lavamos la mano, o sino con alcohol, aquí tengo mi botella también, porque me da miedo que nuevamente pueda tener" (E012).

la forma de transmisión y prevención de esta enfermedad. Ello podría ser debido a que en pocas ocasiones se ha informado sobre esta enfermedad a los pacientes o familiares y, si se hizo, las circunstancias en las que se dieron no habrían sido las más favorables para la captación de la información, debido al drama familiar que vivían los afectados. Además, para producir un cambio en los conoci- mientos a largo plazo se requiere que los mensajes se ofrezcan de manera persistente en el tiempo. Un lugar en el que esto se puede realizar son las instituciones educativas. Ejemplos de ello se encuentran en los trabajos de Sanmartino et al. (2012) y Torres et al. (2015), quienes trabajaron con estudiantes y lograron un aumento y persistencia de conocimientos sobre medidas preventivas 
para la enfermedad de Chagas y conceptos básicos de salud bucal, respectivamente.

En muchos estudios sobre EQ, el impacto de la enfermedad sobre la salud del paciente se ha relacionado principalmente a los signos clínicos que presentaban. Sin embargo, no se toman en cuenta otros aspectos como los relacionados al impacto psicológico. En la investigación realizada se recogió el sentir de los afectados (pacientes y familiares), demostrando que no solo el bienestar físico se ve afectado, sino también el mental y el social. En los entrevistados se observó desde temor a los animales o a comer ciertos alimentos, la tensión al recordar lo vivido, y hasta el rechazo a asistir a los centros hospitalarios por los traumas ocasionados a lo largo de la asistencia para el tratamiento y recuperación.

La enfermedad en una persona supone siempre una situación de crisis y un deterioro de la calidad de vida para el paciente y su familia desde el punto de vista físico y también sicológico (Chahed et al., 2016). Tiene como consecuencias la disminución de la autoestima, la aparición o el aumento de la ansiedad y depresión, el aumento de la sensibilidad al rechazo y la reducción de los intentos de implicarse en actividades usuales de la vida cotidiana; además de verse afectada la dinámica de las relaciones personales intrafamiliares, que cuando la enfermedad es crónica se potencian; también existe la perturbación social, que incluso puede conducir a discordias familiares a menudo relacionadas con problemas económicos (Rodríguez et al., 1993; Caro, 2014). En este contexto, el servicio de salud no solo debe curar al paciente incidiendo sobre el agente etiológico, sino debe preocuparse también por ofrecer un soporte psicológico que permita al paciente y familiares enfrentar la situación de una manera más optimista (Rodríguez et al., 1993).
El conocimiento de la enfermedad y la manera de enfrentarla es de suma importancia debido a que ello evitaría que los familiares opten por alternativas no convencionales. Morales (2012) menciona que en zonas andinas y rurales es común recurrir al uso de la medicina alternativa para enfrentar enfermedades desconocidas. Sin embargo, esto conlleva a que se retrase el tratamiento y curación de las personas, lo que disminuye la probabilidad de sobrevivencia.

El asumir la responsabilidad del tratamiento de la EQ conlleva a la alteración de la tranquilidad y dinámica familiar, el alejamiento del hogar que a su vez se asocia a la necesidad de invertir recursos para viaje, estadía y alimentación. Aunque en la mayoría de los casos el costo de la intervención quirúrgica y parte del tratamiento fue asumido por un seguro estatal, ello no cubría la totalidad del costo, por lo que los padres tenían que buscar recursos económicos extras para solventar parte de las medicinas. Esto supone un alto impacto en el núcleo familiar teniendo en cuenta que los entrevistados, según el INEI, provenían de zonas de alta pobreza (7.9 a $65.9 \%$ ), como son los distritos rurales y semirurales de la provincia de Huancayo, entre ellos Viques, Huayucachi y Chilca (INEI, 2008).

La experiencia con la enfermedad y la adopción de conocimientos inadecuados ha llevado a los familiares de los pacientes a cambiar hábitos de consumo de alimentos en forma errónea, como es el retiro de las vísceras, especialmente hígado, de la canasta familiar. Gonzáles et al. (2015) indican que este alimento debe ser incluido en la dieta en la infancia por su elevado contenido en hierro, ya que es una buena herramienta para la prevención de anemias ferropénicas. Teniendo en cuenta que los índices de anemia en niños en la sierra son de $52.8 \%$ y en la región Junín de 62.6\% (INEI, 2014), evitar el consumo de esta víscera es una práctica inadecuada. 
En todo programa de prevención y control de la EQ, elevar el nivel educativo resulta ser un eje central y debe de estar especialmente dirigido a evitar la alimentación de los perros con vísceras crudas infectadas (Larrieu y Zanini, 2012). En el Perú, esta situación es un reto, entre otros factores, por el alto porcentaje de analfabetismo en las zonas rurales del país (19.5\% de la población mayor de 15 años), según el Censo Nacional del 2007. Sin embargo, podría hacerse uso de otras herramientas para educar a la población analfabeta como, por ejemplo, el empleo de fotografías, recursos audiovisuales, esquemas del ciclo biológico, entre otros, con el fin de no dejar en el olvido a este sector de la población.

\section{Conclusiones}

- Persisten las deficiencias en conocimientos y percepciones con relación a equinococosis quística en familias con antecedentes de la enfermedad.

- La equinococosis quística no solo impacta sobre la salud del paciente infectado, sino en toda la familia. Además, este puede ser emocional, económico y alterar la dinámica familiar.

- Existen deficiencias en la calidad y calidez del trato brindado a los familiares por parte de los profesionales de la salud, lo cual ahonda la crisis emocional de los mismos.

- El desconocimiento de la forma de transmisión de la enfermedad favoreció la adopción de prácticas incorrectas como evitar el consumo de vísceras y el temor a la crianza de animales.

\section{Literatura Citada}

1. Acha P, Szyfres B. 2003. Zoonosis y enfermedades transmisibles comunes al hombre y los animales. Vol III. Parasitosis. $3^{\text {ra }}$ ed. Washington DC: Organización Panamericana de la Salud. $413 \mathrm{p}$.
2. Aybar M, Hernández H, Falcón N. 2012. Equinococosis quística humana autóctona en zona urbana diagnosticada en un hospital de niños en Lima-Perú. 1998-2010. Una Salud. Revista Sapuvet Salud Pública 3(2): 15-28.

3. Cardona GA, Carmena D. 2013. A review of the global prevalence, molecular epidemiology and economics of cystic echinococcosis in production animals. Vet Parasitol 192: 10-32. doi: 10.1016/j.vetpar.2012.09.027

4. Carmena D, Cardona GA. 2013. Canine echinococcosis: global epidemiology and genotypic diversity. Acta Trop 128: 441-460. doi: 10.1016/j.actatropica.2013.08.002

5. Caro D. 2014. Impacto económico de las enfermedades crónicas. Tesis de Ingeniero Comercial. Santiago de Chile: Universidad de Chile. $135 \mathrm{p}$.

6. Chahed MK, Bellali H, Jemaa SB, Bellaj T. 2016. Psychological and psychosocial consequences of zoonotic cutaneous leishmaniasis among women in Tunisia: preliminary findings from an exploratory study. PLoS Negl Trop Dis 10(10): e0005090. doi: 10.1371/ journal.pntd.0005090

7. Fano P, Hernández H, Falcón $N$. 2014. Costos de la atención de pacientes con equinococosis quística humana en un hospital de niños en Lima-Perú, periodo 2006-2010. Salud Tecnol Vet 2(1): 63-70.

8. Gonzáles E, Huamán-Espino L, Gutiérrez C, Aparco J, Pillaca J. 2015. Caracterización de la anemia en niños menores de cinco años de zonas urbanas de Huancavelica y Ucayali en el Perú. Rev Perú Med Exp Salud Pública 32: 431-439.

9. [INEI] Instituto Nacional de Estadística e Informática. 2008. Censos Nacionales 2007. XI de Población y VI de Vivienda. Lima: INEI. [Internet]. Disponible en: http://censos.inei.gob.pe/ cpv2007/tabulados/ 
10. [INEI] Instituto Nacional de Estadística e Informática. 2014. Encuesta Demográfica y de Salud Familiar 2013. Lima: INEI. [Internet]. Disponible en: https://www.inei.gob.pe/media/ MenuRecursivo/publicaciones_digitales/ Est/Lib1151/index.html

11. Irabedra P, Salvatella R. 2010. El proyecto Subregional Cono Sur de Control y Vigilancia de la Hidatidosis. Rev Peru Med Exp Salud Pública 27: 598-603.

12. Jiménez, S, Pérez A, Juste R, Quiñones C. 2004. Diecisiete años de programa control de la hidatidosis en la Rioja: resultados y valoración económica. Bol Epidemiol 196: 1415-1418.

13. Larrieu E, Belloto A, Arambulo P, Tamayo, H. 2004. Echinococcosis quística: epidemiología y control en América del Sur. Parasitol Latinoam 59: 8289. doi: 10.4067/S0717-77122004000100018

14. Larrieu E, Zanini F. 2012. Critical analysis of cysticechinococcosis control programs and praziquantel use in South America, 1974-2010. Rev Panam Salud Pública 31: 81-87.

15. Mandal S, Mandal MD. 2012. Human cystic echinococcosis: epidemiologic, zoonotic, clinical, diagnostic and therapeutic aspects. Asian Pac J Trop Med 5: 253-260. doi: 10.1016/S19957645(12)60035-2

16. Merino V, Falcón $N$, Morel $N$, González G. 2017. Detección de coproantígenos de Echinococcus granulosus en canes de trabajadores de camales y comercializadores de vísceras en Lima metropolitana. Rev Panam Salud Pública 41: e10.

17. Morales R. 2012. Curanderos y académicos: una experiencia en Trujillo (1994 y 1995). Pueblo Continente 23(1): 14-17.

18. Moro P, McDonald J, Gilman R.H, Silva B, Verastegui M, Malqui V, Lescano $G$, et al. 1997. Epidemiology of Echinococcus granulosus infection in the central Peruvian Andes. Bull World Health Organ 75: 553-561.
19. Moro PL, Cavero CA, Tambini M, Briceño Y, Jiménez R, Cabrera $L$. 2008. Prácticas, conocimientos y actitudes sobre la hidatidosis humana en poblaciones procedentes de zonas endémicas. Rev Gastroenterol Perú 28: 43-49.

20. Pérez C. 2007. Proyecto de control de hidatidosis en el Perú por vigilancia epidemiológica. Tesis Doctoral.Lima:Univ. Nacional Mayor de San Marcos. 144 p.

21. Rafael A, Ramos W, Peralta J, Rojas L, Montesinos E, Ortega-Loayza A. 2008. Hidatidosis pulmonar en un hospital de Lima, Perú: experiencia en 113 pacientes. Rev Peru Med Exp Salud Publica 25: 285-289.

22. Reyes MM, Taramona CP, SaireMendoza M, Gavidia CM, Barron E, Boufana B, Craig PS, et al. 2012. Human and canine echinococcosis infection in informal, unlicensed abattoirs in Lima, Peru. PLoS Negl Trop Dis 6: e1462. doi: 10.1371/journal.pntd.0001462

23. Rodríguez J, Pastor M, López S. 1993. Afrontamiento, apoyo social, calidad de vida y enfermedad. Psicothema 5(Supl): 349-372.

24. Rojas K. 2012. Características epidemiológicas de pacientes diagnosticados con hidatidosis en el Hospital María Auxiliadora: periodo 2002-2009. Tesis de Médico Veterinario Zootecnista. Lima: Univ. Peruana Cayetano Heredia. 26 p.

25. Rubio C, Mип̃oz MA, Fuster $C$, Carmona M, Permanyer J, Durán FJ. 2012. Hidatidosis hepática. EuroEco 3(1): 1-5. [Internet]. Disponible en: http:/ /www.euroeco.org/vol3 num1_marzo2012/pdf/01_05.pdf

26. Sanmartino $M$, Mengascini $A$, Menegaz A, Mordeglia C, Ceccarelli S. 2012. Miradas caleidoscópicas sobre el Chagas. Una experiencia educativa en el Museo de La Plata. Revista Eureka sobre Enseñanza y Divulgación de las Ciencias 9: 265-273.

27. Santivañez SJ, Naquira C, Gavidia CM, Tello L, Hernandez, E, Brunetti E, Kachani M, et al. 2010. Factores domiciliarios asociados con la presencia 
de hidatidosis humana en tres comunidades rurales de Junín, Perú. Rev Peru Med Exp Salud Pública 27: 498-505.

28. Torres L, Pérez YE, Pascual D, Noa Y. 2015. Modificación de conocimientos sobre salud bucal en adolescentes de la Secundaria Básica «Orlando Fernández Badell». MediSan 19: 366-374.

29. Velarde PH. 2002. Situación del tratamiento quirúrgico del quiste hidatídico hepático en el Hospital Nacional Arzobispo Loayza, enero 1990 - abril 2000. Tesis de Especialidad. Lima: Univ. Nacional Mayor de San Marcos. 39 p.
30. Venegas J, Espinoza S, Sánchez G. 2014. Estimación del impacto económico de la equinococosis quística en Chile y análisis de las posibles causas que han dificultado su erradicación. Rev Med Chile 142: 1023-1033. doi: 10.4067/ S0034-98872014000800010

31. Zúñiga E. 2014. Características epidemiológicas de pacientes diagnosticados con equinococosis quística humana en el Hospital Nacional Cayetano Heredia, periodo 2008-2012. Tesis de Médico Veterinario Zootecnista. Lima: Univ. Peruana Cayetano Heredia. 21 p. 